
July 1936

\title{
DISTRIBUTION OF THE ENERGY IN THE EXTREME ULTRAVIOLET OF THE SOLAR SPECTRUM
}

\author{
By W. W. Coblentz and R. Stair
}

ABSTRACT

Supplementing the data given in a previous publication (NBS Research Paper RP877), the present paper gives further calculations and graphs showing the distribution of energy in the extreme ultraviolet of the solar spectrum, for various air masses traversed by the rays, at a sea-level station (Washington); also the spectral-energy distribution of solar radiation outside the earth's atmosphere, showing that there is no similarity with the radiation of a black body at $6,000^{\circ} \mathrm{K}$, which is sometimes used in calculating the ultraviolet solar spectral energy.

The second part of the paper describes progress made in assembling apparatus for measuring the spectral quality and total intensity of ultraviolet solar radiation by means of a photoelectric cell and filter radiometer transported aloft in sounding balloons.

I. Introduction

II. Distribution of energy in the extreme ultraviolet of the solar spectrum, within and outside of the earth's atmosphere

1. Correlation of ultraviolet and total solar radiation

III. A meter for determining the ultraviolet solar spectral-energy distribution by means of sounding balloons.

IV. References and notes_.

\section{INTRODUCTION}

In a previous communication $[1]^{1}$ a photoelectric cell and filter radiometer was described for determining the distribution of energy in the extreme ultraviolet of the solar spectrum. Data were given on the distribution of energy in the extreme ultraviolet for various solar altitudes, and the shape of the solar spectral-energy curve outside the earth's atmosphere was discussed. It was shown that the common procedure of calculating the spectral-energy distribution (in the ultraviolet), on the assumption that the radiation is similar to that of a black body at a temperature of $6,000^{\circ} \mathrm{K}$, is untenable because spectroradiometric measurements indicate that there is an abrupt and continuous drop in the solar spectral-energy curve, of wave lengths shorter than $3000 \mathrm{~A}$, outside the earth's atmosphere.

The need having arisen, in the meantime, for more specific information on this subject, in connection with questions on heliotherapy, the object of the present paper is (a) to present further data on the distribution of energy in the extreme ultraviolet of the solar spectrum

\footnotetext{
${ }^{1}$ Numbers in brackets refer to references and notes at the end of this paper.
} 
within and outside the earth's atmosphere, and (b) to give an outline of a method for determining the ultraviolet solar spectral-energy distribution in the stratosphere by means of a photoelectric cell (of special design), filters and auxiliary apparatus, transported to the highest elevations attainable by means of sounding balloons.

This involves the problem of transmitting certain radio signals from the balloon to the ground. For this purpose the writers had the choice of two methods: (a) the newly designed, mechanically controlled, signaling mechanism of Curtiss and Astin [2], or (b) an adaptation of an early type of audion bulb which, when evacuated to a few millimeters gas pressure, transmits a pulsating current that changes in frequency with change in current in the input circuit [3].

As originally used with a photoelectric cell, the signal was received as a change in pitch of the sound in a telephone receiver [3]. For the present purpose this audio signal is used to modulate a transmitted radio wave. Hence, since the radio signals from the photoelectric apparatus, transported by a sounding balloon, are to be received by the Radio Section of the Bureau, the latter method of radio transmission was adopted.

Fortunately, commercial radio tubes are now available ${ }^{2}$ that can be operated to transform feeble photoelectric currents into audio signals suitable to meet our needs. The general hookup of this apparatus is described in section III.

Calculations of intensities to be expected at high altitudes [1], and ground tests of assembled apparatus, indicate that important data on spectral quality (spectral-energy distribution) and total intensity of ultraviolet solar radiation are to be expected from measurements in the stratosphere.

The delay in making field tests is owing to our inability to secure photoelectric cells of special design to meet our requirements.

\section{DISTRIBUTION OF ENERGY IN THE EXTREME ULTRA- VIOLET OF THE SOLAR SPECTRUM, WITHIN AND OUTSIDE OF THE EARTH'S ATMOSPHERE}

In a previous paper [1] data were given on the ultraviolet solar spectral-energy distribution in relative units, but no attempt was made to correlate the various spectral-energy curves for different air masses traversed by the solar rays.

From the average values of the total ultraviolet solar radiation observed through various air masses (solar altitudes) it is possible to obtain factors that give the heights of the various spectral-energy curves relative to each other. In figure 1 are depicted the spectralenergy curves based upon the data previously published [1].

Judged by the closeness with which the data fall upon the isochromatic curves (not published) in which the ordinates were plotted on the logarithmic scale, as commonly employed, the measurements are in good agreement.

As noted in the previous paper [1] the use of two photoelectric cells (differing greatly in spectral response) and four filters gives eight conditions for establishing the shape of the spectral-energy

\footnotetext{
2 The writers are indebted to F. W. Dunmore for calling attention to this newly developed radio tube, which had escaped their notice, although a description of the device was published some time ago [4].
} 


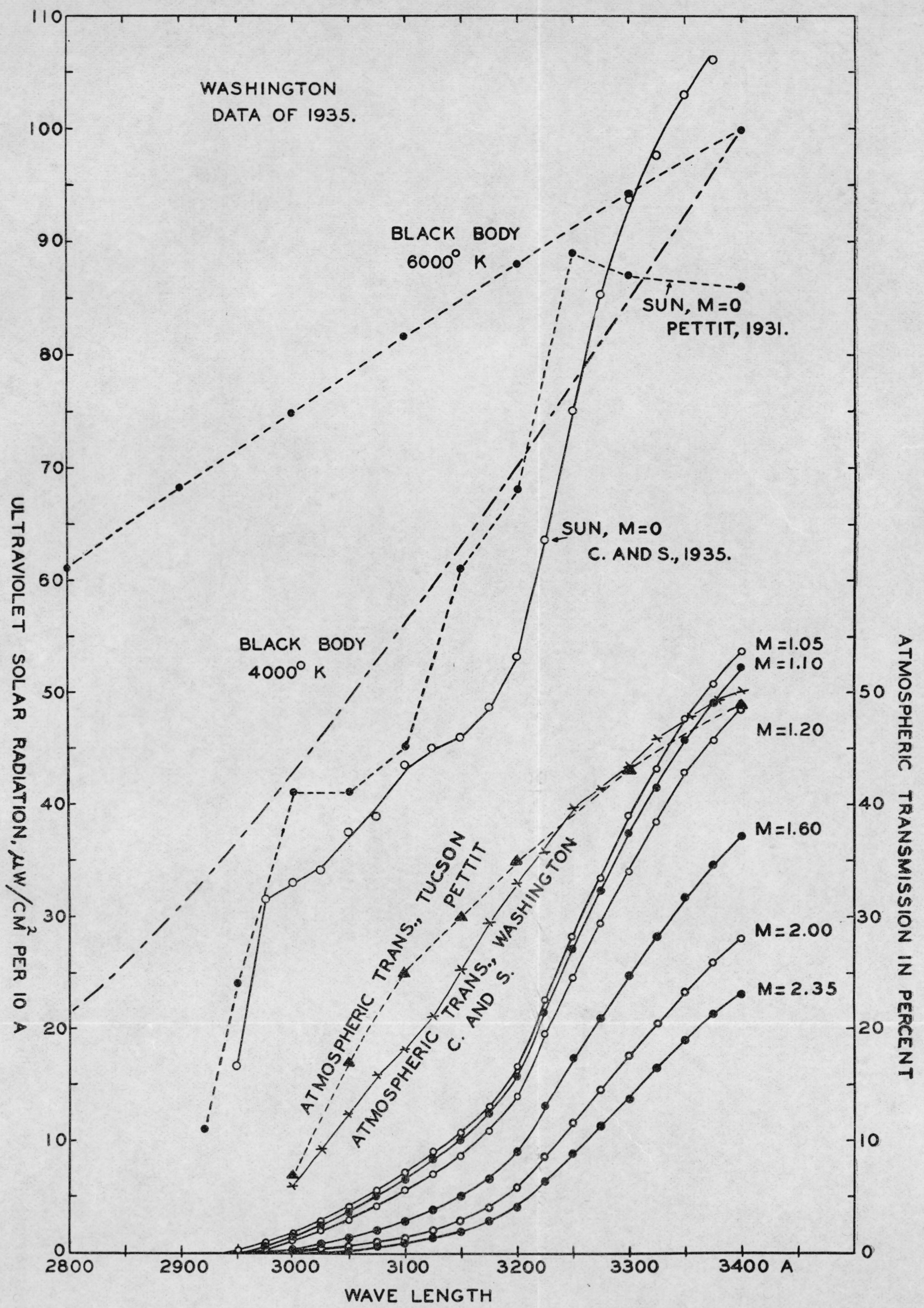

FIGURE 1.-Distribution of energy in the extreme ultraviolet of the solar spectrum, within and outside the earth's atmosphere; also atmospheric transmission curves. 
curve for any given air mass traversed by the solar rays. From this it would appear that this good agreement is real and not the result of a fortuitous combination of factors including (a) the spectral responses of the photoelectric cells, (b) the spectral transmissions of the filters, and (c) the observations of the integrated ultraviolet transmissions through the various filters.

In figure 1 is given the ultraviolet solar spectral-energy distribution outside the earth's atmosphere as deduced by Pettit [5] and by the writers. As noted in the previous paper [1], in both cases the spectral intensities show a continuous drop with decrease in wave length.

In this same illustration is given also the ultraviolet spectralenergy distribution of a black body at $4,000^{\circ} \mathrm{K}$ and at $6,000^{\circ} \mathrm{K}$, respectively. The spectral-energy distribution of a black body at $4,000^{\circ} \mathrm{K}$ represents most closely the observed (extrapolated) data. It, therefore, seems incorrect to compute the ultraviolet solar spectralenergy distribution on the assumption that the radiation is similar to that of a black body at $6,000^{\circ} \mathrm{K}[11,12]$.

The atmospheric transmission curves as deduced from our spectralenergy curves are somewhat lower than observed by Pettit [5] as is to be expected, seeing that the data refer to two stations differing about 2,000 feet in elevation.

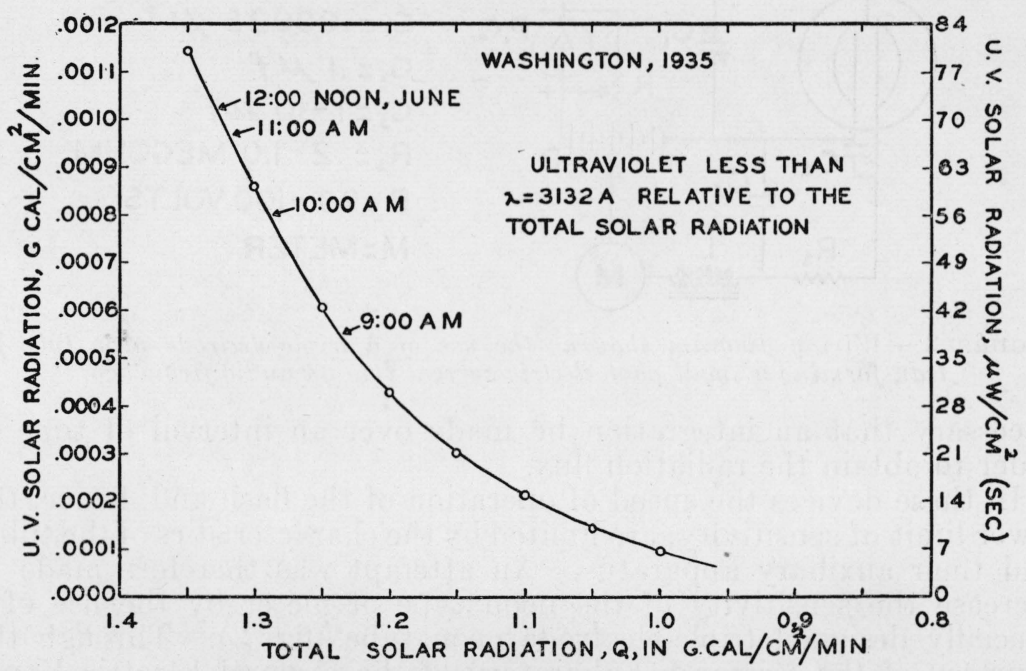

FIGURE 2.

\section{CORRELATION OF ULTRAVIOLET AND TOTAL SOLAR RADIATION}

In view of the fact that in heliotherapy the patient is usually exposed to the total (unfiltered) radiation from the sun, in figure 2 is shown the ultraviolet of wave lengths shorter than and including $3132 \mathrm{~A}$, relative to the total incident solar radiation, in gram calories per square centimeter per minute. The total radiation data are average values, for Washington, taken from the Smithsonian Meteorological Tables.

For a low sun (a long air mass traversed) the spectral quality is different from, and the total intensity is much lower than, that observed at high solar elevations. 


\section{A METER FOR DETERMINING THE ULTRAVIOLET SOLAR SPECTRAL-ENERGY DISTRIBUTION BY MEANS OF SOUNDING BALLOONS}

Various types of direct-current amplifiers have been designed and made to work quite satisfactorily $[6,7,8]$ in the measurement of small currents, such as are produced by photoelectric cells used in the evaluation of short-wave-length ultraviolet solar radiation. In all cases, these devices require the attention of an expert operator to keep the voltage and zero of the scale reading properly adjusted. Hence, they are not suitable for operating automatic-recording instruments, or for the transmission of signals by radio from sounding balloons.

An ultraviolet meter which makes use of a glow relay tube has been described by Rentschler [9], and a somewhat similar meter which uses a neon-flash lamp has been described by Taylor [8]. While these instruments are very useful, they have their limitations in that for accurate reproduction their speed of operation is limited to a small number of impulses ("clicks" or "flashes") per minute. Hence it is
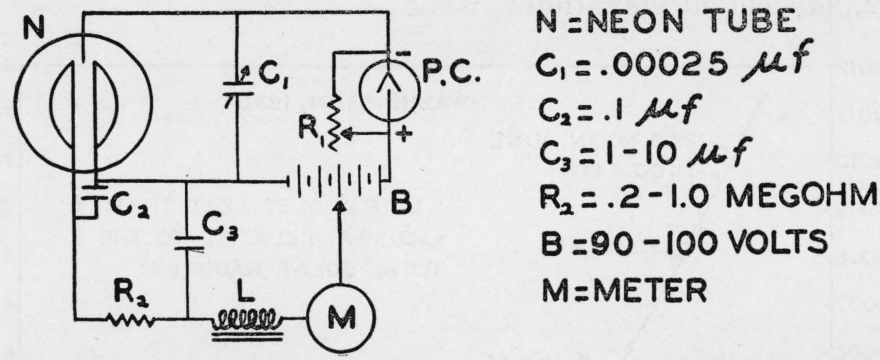

FIGURE 3.-Wiring diagram showing the use of a triple-electrode neon tube for transforming a small photoelectric current into an audio frequency.

necessary that an integration be made over an interval of time in order to obtain the radiation flux.

In these devices the speed of operation of the flash and, hence, the lower limit of sensitivity, are limited by the characteristics of the tubes and their auxiliary apparatus. An attempt was therefore made to increase the sensitivity of the neon type of meter by the use of a specially designed triple-electrode neon tube (fig. 3). Through the courtesy of the Research Laboratory of the General Electric Vapor Lamp Co., Hoboken, N. J., several triple-electrode neon tubes were constructed. While they are much superior to the ordinary neon tubes having two electrodes, they have several defects which prevent their use in ultraviolet meters for precision measurements. There is a leakage in these tubes that must be counterbalanced by a resistance in parallel with the photoelectric cell. Moreover, the breakdown and recovery voltages are not absolutely constant. Although much more sensitive than neon tubes having two electrodes, the sensitivity is insufficient to give the high audio frequency desired when activated by small photoelectric currents, and operated in connection with radio apparatus and frequency meters.

It is to be noted that the triple-electrode neon tube may find valuable applications in the operation of relays without the use of auxiliary radio amplifying apparatus. 
Consideration was then given to other methods of producing an audio-frequency signal, including the use of a leaky triode previously used by one of the writers [3] for the production of a change in pitch of the sound in a telephone receiver, when used in connection with a photoelectric cell. In this connection our attention was called to the use, by Herold [4], of a standard radio tube (type 57) in place of a neon tube to produce an audio frequency.

For our work a similar type (1A6) tube was chosen because of its low filament current and voltage. This tube is a stable oscillator and has a high grid-circuit resistance (partly due to the location of the grid at the top of the tube), and, hence, provides an extremely sensitive means for measuring low photoelectric currents. The complete instrument (see fig. 4) is about one hundred times as sensitive as could be made by using the special neon tubes, and should be practical for measuring currents as low as about $10^{-12}$ or $10^{-13}$ ampere.

In operation, the condenser $\mathbf{C}$ is adjusted to the proper capacity to give a suitable audio signal when the photoelectric cell is illuminated. On covering the cell the frequency of the signal generated by the tube decreases to one or two cycles per second.

Only the generator stage is illustrated in figure 4. The output from this unit may be coupled directly into the transmitting oscillator tube of a radio transmitter on an unmanned balloon. If, however, the meter is to be used at a ground station, additional stages of audio-
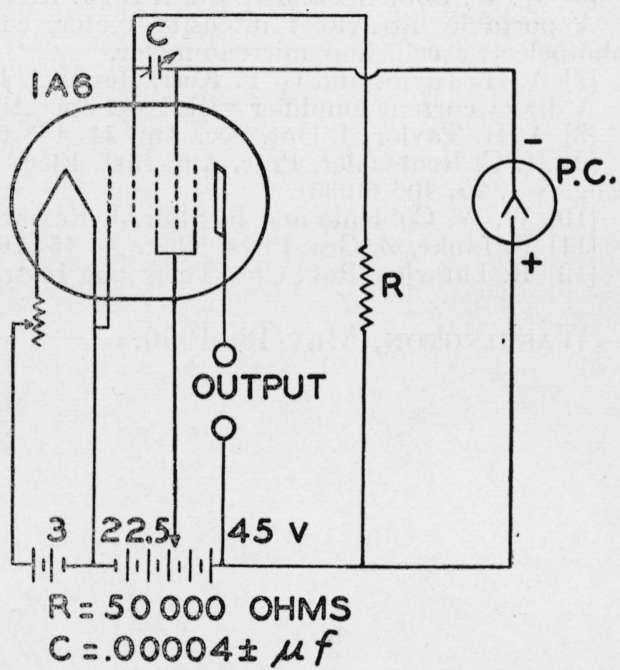

Figure 4.-Wiring diagram of the photoelectric audio-frequency generator for modulating the carrier wave in the radio transmission of signals.

frequency amplification must be added to supply sufficient power to operate a frequency meter or an automatic frequency recorder.

This instrument may be calibrated against a previously standardized meter, or directly against an ultraviolet standard of radiation [10], as is done with the ordinary type of balanced amplifier photoelectric meter [6]. The calibration of the frequency scale is made in terms of microwatts per square centimeter, for example, for a certain band of radiation in sunlight. The radiation intensity may be read directly from a calibrated frequency meter or automatically recorded with suitable apparatus.

However, the primary purpose of this investigation was the development of means of transforming small photoelectric currents into suitable pulses for automatic transmission by radio from small sounding balloons released into the upper atmosphere. When coupled into the oscillator circuit of a small transmitter this method appears to be ideal for transmitting data on the intensity and spectral quality of the ultraviolet solar radiation in terms of an audio-frequency modulation of the carrier wave. 


\section{REFERENCES AND NOTES}

[Bracketed numbers are the designating literature reference numbers as cited in the text of this paper]

The following publications of the National Bureau of Standards are obtainable only through the Superintendent of Documents, Washington, D. C., prepaid, at the prices indicated.

[1] W. W. Coblentz and R. Stair, J. Research NBS 16, 315 (1936) RP877 (10c).

[2] L. F. Curtiss and A. V. Astin, Aeronaut. Sci. 3, 35 (1935).

[3] W. W. Coblentz, BS Sci. Papers 16, 635 (1920) S398 (10c); Misc. Pub. NBS 46 , p. 138 (1921).

Thermal-radiophonic devices for secret signaling.

[4] E. W. Herold, Proc. Inst. Radio Eng. R3, 1200 (1935).

Negative resistance and devices for obtaining it.

[5] E. Pettit, Astrophys. J. 75, 185 (1932); Pub. Astronom. Soc. Pacific 47, No. 280 (December 1935).

[6] W. W. Coblentz and R. Stair, BS J. Research 12, 231 (1934) RP647 (5c).

A portable ultraviolet intensity meter, consisting of a balanced amplifier, photoelectric cell, and microammeter.

[7] A. H. Taylor and G. P. Kerr, Rev. Sci. Instr. 4, 28 (1933).

A direct current amplifier with good operating characteristics.

[8] A. H. Taylor, J. Opt. Soc. Am. 24, 183 (1934).

[9] H. C. Rentschler, Proc. Am. Inst. Elec. Eng. 49, 113 (1930); Trans. Illum. Eng. Soc. 25, 406 (1930).

[10] W. W. Coblentz and R. Stair, J. Research NBS 16, 83 (1936) RP858 (5c).

[11] F. Linke, Z. Ges. Phys. Therapie 45, 169 (1933).

[12] R. Latarjet, Rev. Opt. Théorique Instr. 14, 398 (1935).

Washington, May 18, 1936. 